\title{
Health and social care professionals' attitudes to interprofessional working and interprofessional education: A literature review
}

\begin{abstract}
The healthcare setting is a rich learning environment for students to experience interprofessional working (IPW) and interprofessional education (IPE). However, opportunities for IPE are limited, and student experiences of effective IPW are varied. This raises the question of how IPW and IPE are valued by health or social care professionals. A search of the literature was carried out to identify studies of health and social care staff attitudes to IPW and IPE. This review provides a summary of the main factors found to influence attitudes and the strengths and limitations of these studies. Professional background and prior IPE experience were identified as the influencing factors for which there is most evidence. The main limitations of the studies accessed included a focus on the value of IPE for staff, as opposed to students, and a limited number of studies considering the relationship between attitudes to IPW and the value placed on IPE. It is important that health and social care professionals lead by example by working collaboratively and providing students with opportunities for IPE. Identifying the variables influencing attitudes to IPW and IPE may assist in improving IPW and experiences of IPE for students learning in the healthcare setting.
\end{abstract}




\section{Introduction}

The integration of health and social care in the United Kingdom (Health and Social Care Act, 2012) is one of the most recent drivers for effective interprofessional working (IPW) and for providing students with opportunities for interprofessional education (IPE; Barr \& Low, 2013; General Medical Council, 2009; Health and Social Care Professions Council, 2009; Nursing and Midwifery Council, 2010; World Health Organisation, 2010). This integration combines the services of health boards and local councils to reduce the numbers of unecessary admissions to hospitals by providing a more coordinated, cost effective approach to the provision of quality health and social care. Integration has implications for the way that health and social care teams collaborate and effective IPW is key to its success (Association of the Directors of Social Work, 2013).

Although further high quality research is required to support the impact of IPE and IPW on patient care (Reeves, Perrier, Goldman, Freeth, \& Zwarenstein, 2013; Zwarenstein, Goldman, \& Reeves, 2009), previous inquiries have highlighted the impact that ineffective IPW can have on patient safety (Kennedy, 2001; Laming, 2003). Twelve years after the Bristol Infirmary Heart Inquiry (Kennedy, 2001), the Mid Staffordshire Report (Francis, 2013) highlighted that little progress had been made in changing the damaging, target driven culture of the health service. Francis (2013) recommended that this culture needed to change to put the patient first.

Time pressures, limited resources, lack of understanding of roles and responsibilities, competing priorities, and access to other professionals prevent effective IPW between professional groups (Bailey, Jones, \& Way, 2006; Braithwaite et al., 2012; Hughes \& McCann, 2003; Kvarnström, 2008; Larkin \& Callaghan, 2005; 
Snelgrove \& Hughes, 2000). Healthcare teams are capable of improvising and developing strategies to work around such barriers (Baxter \& Brumfit, 2008; Lingard et al., 2012). However, ineffective IPW continues to affect the quality of care provided by health and social care teams (Laming, 2003).

As part of the practice placement agreements between health boards and higher education institutes, health and social care students spend a proportion of time learning within healthcare settings. Francis (2013) highlighted how vital it is that students are exposed to positive experiences in these settings:

"Good practical training should only be given where there is good clinical care. Absence of care to that standard will mean that training is deficient. Therefore, there is an inextricable link between the two that no organisation responsible for the provision, supervision or regulation of education can properly ignore" (p.60).

Effective IPW is essential for maintaining quality care and improving patient safety (Francis, 2013; Gordon, 2012; Laming, 2003; The Joint Commission, 2013). The healthcare setting can be an invaluable opportunity to learn with students from other professions and to gain first-hand experience of how health and social care teams work (O'Carroll, Braid, Ker, \& Jackson 2012; O'Carroll, Smith, Collinson, Jackson, \& Ker, 2013). However, opportunities for IPE and students' experiences of IPW within the healthcare setting can be varied and limited (Pollard, Miers, \& Rickaby, 2012; Stew, 2005). The logistical difficulties of coordinating student timetables, negative attitudes and uncertainty of the value of IPE have been identified as reasons why support for IPE within the academic setting falters (Curran, Sharpe \& Forristall, 2007; Freeth, Hammick, Reeves, Koppel, \& Barr, 2005; Rees \& Johnson, 2007). These are challenges that have been identified within the academic setting, but it is possible that similar ambivalent attitudes exist with the healthcare setting. 
The relationship between attitudes and behaviour is considered within the field of social psychology (Azjen \& Fishbein,1977; Bem, 1970; Festinger, 1957). Kraus (1995) argued that there is significant evidence that attitudes can predict behaviour. It can be argued that investigating the attitudes of health and social care teams to IPW and IPE, may provide an understanding of the value that is placed on IPW and IPE for students learning in healthcare settings. It is important that these issues are studied directly, within a relevant context, and from the perspective of staff working within these settings. This literature review provides a summary and critique of the existing literature related to two main constructs:

- The attitudes of health and social care professionals, working in healthcare settings, to IPW

- The attitudes of health and social care professionals, working in healthcare settings, to IPE for students

\section{Methods}

A systematic search was carried out using The Cochrane Database of Systematic Reviews, Education Resources Information Centre (ERIC), MEDLINE, and the Cumulative Index to Nursing and Allied Health Literature (CINAHL). Relevant papers were also identified from a hand search of the Journal of Interprofessional Care and by interrogating references from relevant papers. As the turn of century was a significant turning point for IPE and IPW (Barr, Helme \& D'Avray, 2011), the search focused on literature published between 2000 and 2014 . Table 1 provides a summary of the search strategy, including the inclusion and exclusion criteria. The terms 'interprofessional,' 'multidisciplinary,' and 'interdisciplinary' were used in combination with the following key words; learning, 
education, working, collaboration, attitudes, staff and healthcare. Boolean operators and truncations were used to allow for variability of terms adopted in the literature.

Table 1. Summary of search strategy

Inclusion criteria $\quad$ Exclusion criteria

Studies measuring and comparing health and social work staff attitudes within hospital and community healthcare environment to IPE and IPW

Studies using quantitative, qualitative or mixed methods

Year 2000 to 2014

Published in peer reviewed journals

Studies written in the English language
Students' attitudes (only) to IPE and IPW

Academic staff attitudes (only) to IPE and IPW

Studies exploring the attitudes of only one professional group

Editorials

Descriptive articles

Opinion pieces

\section{Search terms}

Key words

Interprofessional/multidisciplinary/interdisciplinary learn* and attitude $\$ 1$

Interprofessional/multidisciplinary/interdisciplinary learn* and attitude $\$ 1$ and staff

Interprofessional/multidisciplinary/interdisciplinary education and attitude $\$ 1$ and staff

Interprofessional/multidisciplinary/interdisciplinary work*and attitude $\$ 1$

Interprofessional/multidisciplinary/interdisciplinary work* and healthcare

Interprofessional/multidisciplinary/interdisciplinary collaboration and healthcare

Figure 1 reports on the number of studies included and excluded at different phases of the literature search. On initial identification and screening of titles and abstracts, 59 papers met the inclusion criteria. Thirty five studies were selected after excluding the following: studies within the context of an academic setting; student attitudes to IPW and IPE; and the attitudes of only one professional group. 

through database searching

22 additional records identified through other sources

925 records after duplicates removed

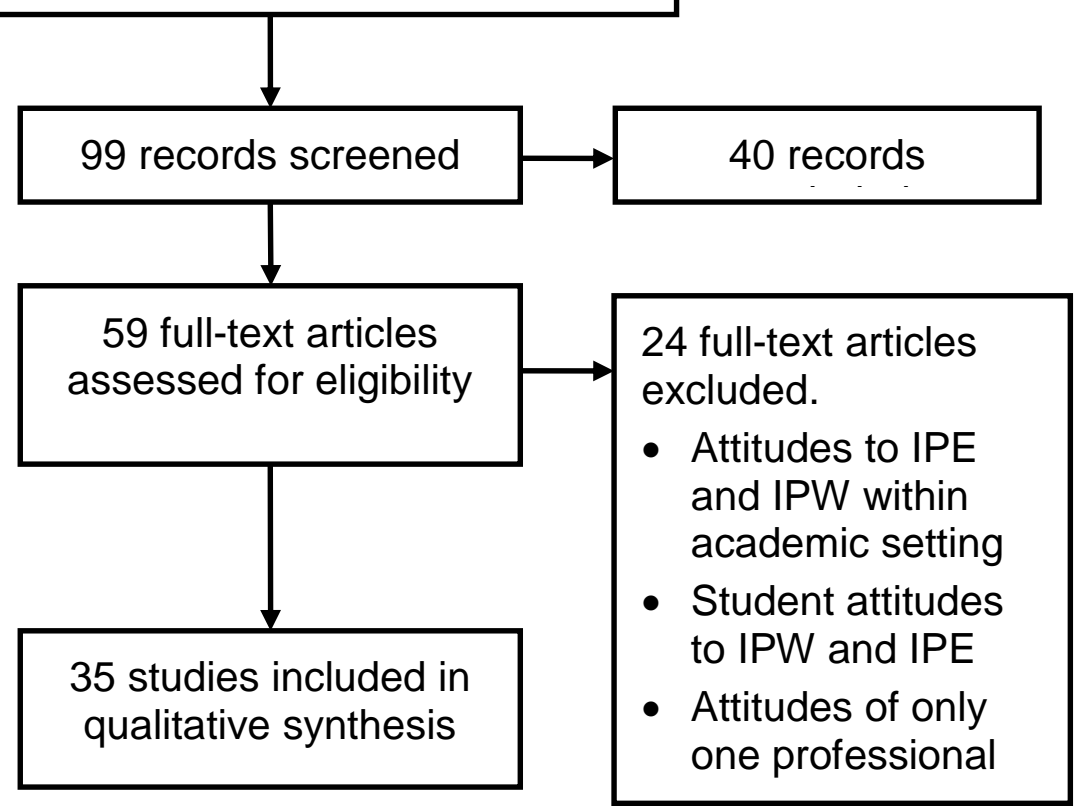

Figure 1. PRISMA flow diagram (Moher, Liberati, Tetzlaff \& Altman, 2009).

The full texts of relevant studies were accessed and interrogated using Hawker, Payne, Kerr, Hardy, and Powell's (2002) framework for appraising research and to reviewing IPE studies where mixed methodologies were used (Ireland, Gibb, \& West, 2008). The three stages for appraising research outlined by Hawker et al. were implemented: assessment of the relevancy of the studies using the inclusion and exclusion critera; extracting data to assist in the process of identifying themes; and scoring the methodological rigor of each study. 


\section{Results}

A total of 35 studies met the inclusion criteria for this review, and no studies were excluded on the basis of poor quality. Fourteen of these studies included health and social care staff as research participants (Table 2). Although four studies included attitudes to IPE and IPW, the study by Baker et al, (2011) was the only study to include health and social care staff attitudes to both IPW and IPE for students within the context of the healthcare setting. Despite these limitations, this review was valuable in identifying two main common themes which included the effect of:

- professional background on attitudes to IPW and IPE

- previous experience of IPE on attitudes to IPW and IPE 
Table 2. Summary of studies included in the review

\begin{tabular}{|c|c|c|c|c|}
\hline Authors & Year & Country & Staff groups included & IPE/IPW focus \\
\hline Abramson \& Mizrahi & 2003 & USA & Health \& social care & IPW \\
\hline Anderson et al. & 2006 & UK & Healthcare & IPE \\
\hline Anderson et al. & 2009 & UK & Academic \& healthcare & IPE \\
\hline Anderson \& Thorpe & 2010 & UK & Academic \& healthcare & IPE \\
\hline Anderson et al. & 2011 & UK & Academic \& healthcare & IPE \\
\hline Bailev et al. & 2006 & Canada & Healthcare & IPW \\
\hline Baker et al & 2011 & Canada & Health \& social care & IPE \& IPW \\
\hline Baxter \& Brumfit & 2008 & UK & Healthcare & IPW \\
\hline Braithwaite et al. & 2012 & Australia & Healthcare & IPE \& IPW \\
\hline Braithwaite et al. & 2013 & Australia & Healthcare & IPE \& IPW \\
\hline Chang et al. & 2009 & R.O.C & Healthcare & IPW \\
\hline Colyer & 2008 & UK & Academic \& healthcare & IPE \\
\hline Costa et al. & 2014 & USA & Healthcare & IPW \\
\hline Egan-Lee et al. & 2011 & Canada & Health \& social care & IPE \\
\hline Gibbon et al. & 2002 & UK & Health \& social care & IPW \\
\hline Herbert et al. & 2007 & Canada & Healthcare & IPW \\
\hline Hughes \& McCann & 2003 & UK & Healthcare & IPW \\
\hline Jové et al. & 2014 & Spain & Healthcare & IPW \\
\hline Kvarnström & 2008 & Sweden & Healthcare & IPW \\
\hline Larkin \& Callaghan & 2005 & UK & Health \& social care & IPW \\
\hline Lindbland et al. & 2006 & Sweden & Healthcare & IPW \\
\hline Lingard et al. & 2012 & Canada & Health \& social care & IPW \\
\hline Matziou al el. & 2014 & Greece & Healthcare & IPW \\
\hline McCray & 2003 & UK & Health \& social care & IPW \\
\hline Piquette et al. & 2009 & Canada & Healthcare & IPW \\
\hline Pollard \& Miers & 2008 & UK & Health \& social care & IPW \\
\hline Pollard et al. & 2012 & UK & Health \& social care & IPE \& IPW \\
\hline Reeves \& Lewin & 2004 & UK & Health \& social care & IPW \\
\hline Reid et al. & 2006 & UK & Healthcare & IPE \\
\hline Rice et al. & 2010 & Canada & Health \& social care & IPW \\
\hline Robben et al. & 2012 & Netherlands & Health \& social care & IPW \\
\hline Russell et al. & 2006 & Canada & Health \& social care & IPW \\
\hline Snelgrove \& Hughes & 2000 & UK & Healthcare & IPW \\
\hline Suter et al. & 2009 & Canada & Healthcare & IPW \\
\hline Wittenberg-Lyles et al. & 2010 & USA & Health \& social care & IPW \\
\hline
\end{tabular}


Effect of Professional Background on Attitudes to IPW and IPE

The effect of professional background on attitudes to IPW and IPE, and differences in attitudes among different professional groups was discussed by a number of studies. Reid, Bruce, Allstaff, and McLernon (2006) measured attitudes to IPW and reported that physicians had less positive attitudes to IPW, in comparison to nurses. Similar professional differences were reported by Chang, Ma, Chiu, Lin, and Lee (2009), with physicians reported to have the least positive attitudes to IPW. In addition, Braithwaite et al., (2012; 2013) found that allied health professionals (AHPs) had more positive attitudes to IPW and IPE than physicians and nurses. These studies were carried out in a number of different countries (Table 2), and their findings highlight that although there may be cultural differences across global healthcare teams, professional background may be a common influence on attitudes.

Professional culture and professional identity. Jové et al., (2014); Rice, Zwarentstein, Conn, Kenaszchuk, Russell and Reeves (2010) and Robben et al., (2012), suggest that professional identity, professional culture and interprofessional hierarchies may influence attitudes to IPW. These researchers reported that healthcare staff often felt that it was unnecessary to collaborate with other professions. Physicians who were found to have negative attitudes to IPW were also found to have a greater perception of their role as main decision makers in the healthcare team (Abramson \& Mizrahi, 2003; Reid et al., 2006; Rice et al., 2010; Russell, Nyhof-Young, Abosh, \& Robinson, 2006). The implications of perceptions of power on attitudes to IPE was discussed by Baker et al., (2011). The results of their study suggested that this perception may have negatively impacted on attitudes to IPE, as physicians were reported to be less engaged in IPE initiatives within the 
healthcare setting, in comparison to nurses and AHPs. These findings suggest that a greater sense of professional identity and professional culture as well as a lack of understanding of roles and responsibilties may influences attitudes to IPW and IPE. This reinforces the important role that IPE may have in helping professionals to develop an appreciation of each other's skills and expertise (Robben et al., 2012).

Differences in the perceived level of IPW. Although there may be some consistency amongst healthcare staff with regards to what they believe is required for effective IPW (Suter et al., 2009), as demonstrated in the work of Chang et al., (2009), Lindbland, Kjellgren, Ring, Maroti, and Serup (2006); and Matziou et al., (2014), professions from the same team can differ in their perceptions of how effective IPW actually is within their team. Similarly, professional differences were reported in their perception of the requirement for IPW in the team (Piquette, Reeves \& Leblanc, 2009). The study by Wittenberg-Lyles, Oliver, Demiris, and Regehr (2010) compared health and social care professionals' perceptions of collaboration from questionnaire responses with their observations of these professionals working together. Wittenberg-Lyles et al. reported differences between staff perceptions of collaboration, which were perceived by staff to be high, and the actual level of collaboration observed within the meetings, which were assessed as more limited. The results of these studies raise the question of how aware health and social care professionals are of effective or ineffective IPW within their own teams and also how much of consensus exists in terms of the perceived requirement for IPW. Effect of Previous Experience of IPE on Attitudes to IPW and IPE

Prior IPE as an influence on attitudes to IPW. IPE as an influence on staff attitudes to IPW was explored in a number of studies (Bailey et al., 2006; Braithwaite et al., 2012; Gibbon et al., 2002; Kvarnström, 2008; Pollard \& Miers, 2008; Pollard et 
al., 2012; Robben et al., 2012). In two studies in particular, IPE interventions provided during undergraduate training were assessed as a positive influence on attitudes to IPW (Pollard \& Miers, 2008; Pollard et al., 2012). As qualified healthcare professionals, staff with experience of IPE in their pre-qualifying training felt more prepared for IPW and had increased self-awareness of positioning in a team, compared to those without prior IPE experience (Pollard \& Miers, 2008). Staff with experience of IPE as students, reflected on varied experiences of IPE and IPW and reported more awareness of barriers to effective IPW such as professional boundaries, hierarchies, and poor communication (Pollard et al., 2012).

Although a history of IPE for staff may improve awareness of roles and responsibilities and attitudes to other professionals, stated behavioural intentions to work in a more interprofessional manner can diminish over time (Bailey et al., 2006; Braithwaite et al., 2013; Robben et al., 2012) or may reinforce perceptions of professions' roles. This reinforcement of perceptions was demonstrated in the study by Braithwaite et al., (2012). Following a series of varied IPE interventions and measurement of attitudes to IPE and IPW over 3 years, there were no significant changes in attitudes to IPE and IPW. However, in relation to their perceptions of the physician as the central role in the team, mean scores increased over the duration of the study, indicating that these perceptions were reinforced over time.

Prior IPE as an influence on attitudes to IPE. Some researchers considered the possible influence that prior experiences of IPE as a learner or as an educator may have on attitudes to IPE (Anderson, Manek, \& Davidson, 2006; Anderson \& Thorpe, 2010; Anderson, Thorpe, \& Hammick, 2011; Egan-Lee, Baker, Tobin, Hollenberg, Dematteo \& Reeves, 2011; Pollard \& Miers, 2008; Pollard et al., 2012; Reid et al., 2006). In their longitudinal study, to determine health and social work 
staff attitudes to IPE and IPW from training to practice, Pollard and Miers (2008), reported that staff who had prior experience of IPE during their training were less positive about IPE as qualified professionals. As previously discussed, Pollard and Miers argued that experiences of IPE as students still influenced their practice, as was evident by their positive attitude to IPW. It is interesting that staff were less positive about IPE than expected, and further investigation into the nature of their IPE experiences as students would be required to explain this result.

Prior IPE experience as an educator and the influence on attitudes to IPE was discussed by Anderson et al., (2006); Anderson, Cox, and Thorpe, (2009); Anderson and Thorpe (2010); Egan-Lee et al., (2011). These studies highlighted that prior uncertainties, doubt and ambiguity related to the value of IPE for students, decreased once staff experienced IPE as a facilitator. This was particularly evident where structured, accredited training and support was given to staff to prepare for facilitating IPE.

Other studied variables found to influence attitudes. The effects of other variables such as professional experience, income, job satisfaction, gender on attitudes were considered briefly by some researchers. According to Chang et al., (2009), staff who were most satisfied with their jobs had more positive attitudes to IPW. Some significant findings related to age, professional experience and attitudes were discussed by Herbert et al., (2007); Matziou et al., (2014); Pollard and Miers, (2008); and Reid et al., (2006). The team's size and structure, staff location and physical access to each other, and the variation in each professionals' operational polices were also considered as possible influences on the ability to deliver effective IPW and IPE (Baxter \& Brumfit, 2008, Larkin \& Callaghan, 2005). Prior experience of IPW was discussed by Jové et al., (2014) who found that those staff who had 
worked collaboratively before were found to have more positive attitudes to IPW. The differences in attitudes between regions and locality of hospitals was also considered as a possible influence on attitudes, although researchers acknowledged that this relationship would need to be further explored (Jové et al., 2014).

\section{Assessment of the quality of the studies}

Hawker et al., (2002) described the assessment of methodological rigor as the third and final stage of systematically reviewing research. Their assessment criteria was used as a guide to assess the quality of the studies for this review. This led to the identification of strengths and limitations which fall into two broad themes: research design and study samples.

Strengths and limitations in research designs. The studies reviewed used a variety of research designs but the most effective design to gain in-depth insight into staff attitudes and an understanding of the challenges in healthcare settings was a mixed method approach (Anderson et al., 2009; Anderson \& Thorpe, 2010; Baxter \& Brumfit, 2008; Lingard et al., 2012; McCray, 2003; Reeves \& Lewin, 2004; Robben et al., 2012; Wittenberg-Lyles et al., 2010). Questionnaires provided data for statistical analysis, which was valuable in measuring influences on attitudes, such as professional background. However, for the studies that only used quantitative methods such as questionnaires, the data did not provide a detailed understanding of specific enablers and barriers to IPW and IPE. There were also missed opportunities by researchers to investigate the influence of prior experience of post qualifying IPE on staff attitudes to IPE and IPW (Pollard et al 2012; Reid et al., 2006). 
Many of the researchers investigated the attitudes of staff within one department or one hospital (Baxter \& Brumfit, 2008; Lingard et al., 2012; McCray, 2003; Snelgrove \& Hughes, 2000; Wittenberg-Lyles et al., 2010) or with teams known to work very closely together (Bailey et al., 2006; Costa, Barg, Asch \& Kahn, 2014; Reeves \& Lewin, 2004; Reid et al., 2006; Robben et al., 2012). Although statistically significant differences between professional groups in terms of the value they placed on IPE were reported in some of the studies (Reid et al., 2006; Robben et al., 2012), it would be difficult to generalise these findings from one department or unit in considering the different systems of working. The context and structure of the team may affect how a team works (Baxter \& Brumfit, 2008; Snelgrove \& Hughes, 2000) and how well established a team is may influence staff attitudes to teamwork (Gibbon et al., 2002; Pollard \& Miers, 2008). Although, a case study approach, as adopted by Baxter and Brumfit (2008) and Egan-Lee et al., (2011) adds to the body of research and builds on the findings from other studies within the field (Stake, 2005; Yin, 2003), investigating a number of different departments and using quantitative and qualitative methods of data collection is essential if the working environment is to be investigated as a variable.

Strengths and limitations in study samples. Sampling techniques, sample size and representativeness of samples were varied across studies, highlighting limitations and strengths for each of the studies. Although use of mixed methods was indentified as a strength in research design, one of the challenges of using mixed methods was maintaining the sample size for the duration of some studies. Anderson et al., (2009); Anderson et al., (2011); and Braithwaite et al., (2012) reported a decrease in sample after the first phase of the research. The transitory nature of teams and high turnover of staff within a healthcare setting may be a 
contributory factor here. This is important to consider for future research and for identifying ways in which sample size can be maintained.

Three of the studies included staff from both a healthcare and academic setting, but representation of different professional groups was unclear in some studies (Anderson et al., 2009; Anderson \& Thorpe, 2010; Baker et al., 2011; Colyer, 2008). Without consideration for the different teaching contexts, it was difficult to identify barriers and enablers to IPW and IPE that may have been specific to each of these contexts or to generalise. For many of the studies, sample demographics, were unspecified, which made it difficult to identify the range of characteristics within the sample groups (Anderson et al., 2009; Anderson \& Thorpe, 2010; Baxter \& Brumfit, 2008; Gibbon et al., 2002; Lindbland et al., 2006; Reeves \& Lewin, 2004; Suter et al., 2009; Wittenberg-Lyles et al., 2010). The studies that were successful in recruiting large samples of staff from a mixture of different mixed professional groups primarily used quantitative methods to collect data (Braithwaite et al., 2012; Chang et al., 2009; Pollard \& Miers, 2008; Reid et al., 2006). Although this approach may have facilitated the inclusion of large samples, by using only quantitative methods the researchers' ability to investigate participants' responses to questionnaires was restricted. A more in-depth investigation of staff perceptions of IPW and IPE was evident where researchers used mixed methods (Anderson et al., 2009; Anderson \& Thorpe, 2010; Robben et al., 2012; Russell et al., 2006; Wittenberg-Lyles et al., 2010), ethnographic approaches to gain an indepth understanding of IPW (Rice et al., 2010) or where researchers compared the attiudes of small samples of different professional groups (Bailey et al., 2006; McCray, 2003; Snelgrove \& Hughes, 2000) 


\section{Discussion}

Two main common themes where identified in relation to the factors effecting attitudes to IPE and IPW from the 35 eligible studies in this review: the effect of professional background on attitudes to IPW and IPE, and the effect of previous experience of IPE on attitudes to IPW and IPE. However, only four of these studies focused on attitudes to both IPE and IPW (Baker et al., 2011; Braithwaite et al., 2012, 2013; Pollard et al., 2012); two of these studies included health and social care staff (Baker et al., 2011; Pollard et al., 2012); and only one study by Baker et al., (2011) focused on health and social care staff attitudes to IPW and to IPE for students learning within a healthcare setting. This highlights the requirement for further research to understand the challenges of IPW and IPE for students within a healthcare setting.

In relation to professional background as an influencing factor, physicians were reported to have the least positive attitudes to IPW in five of the studies (Abramson \& Mizrahi, 2003; Chang et al., 2009; Reid et al., 2006; Rice et al., 2010; Russell et al., 2006). Two of the studies found that AHPs were more positive in their attitudes to IPW and IPE, in comparison to other members of the healthcare team (Braithwaite et al., 2012; 2013). Professional identity and perception of the physician as the main decision maker was recognised as possible effect on these attitudes. This was also verified by Baker et al., (2011) and also linked with physicians lack of engagement with IPE initiatives for students. Boundaries between professionals impacts on the provision of effective healthcare (Powell \& Davies, 2010), and this review highlights the need for further research to investigate how professional boundaries influence attitudes to IPW and IPE. 
Two studies reported IPE during pre-qualifying training as a possible effect on attitudes to IPW as qualified professionals: Pollard and Miers (2008) found that staff had an increased awareness of their positioning in a team; and Pollard et al (2012) reported that staff with prior had a better understanding and awareness of the challenges to effective IPW. This further reinforces the need for further high quality research to assess the impact of different types of IPE interventions on practice (Reeves et al., 2013).

A lack of clarity and understanding around the true concept of IPE and IPW remains in healthcare and education (Reeves, Goldman, Gilbert, Tepper, Silver, Suter and Zwarenstein, 2011) and as reported by Egan-Lee et al., (2006), experience of facilitating IPE can assist with providing this clarity. The positive impact that prior IPE experience as a facilitator can have on attitudes to IPE was also reported in three of the studies in this review (Anderson et al., 2006; Anderson, Cox \& Thorpe 2009; Anderson \& Thorpe 2010). However, as Pollard and Miers (2008) reported, positive attitudes to IPE prior during pre qualifying training may not necessarily continue from training into qualified practice (Pollard \& Miers, 2008). Further research is required to investigate the effect of prior experience of IPE on attitudes to IPW and IPE.

It is acknowledged that although self reporting methods are an effective way of objectively measuring attitudes, there is a risk that research participants may already be ambassadors of IPE or IPW (Anderson et al., 2009; Anderson \& Thorpe, 2010; Robben et al., 2012). Five studies in this review combined qualitative and quantitative methods in their research. Anderson et al., (2009); Anderson and Thorpe, (2010); Robben et al., (2012); Russell et al., (2006); and Wittenberg-Lyles et al., (2010) capitalised on the strengths of these methodologies in their IPE and IPW 
research by using qualitative data to validate and verify quantitative data (Kenaszchuk, Conn, Dainty, McCarthy, Reeves \& Zwarenstein, 2012). Five studies in this review used an ethnographic approach to collecting data (Baxter \& Brumfit, 2008; Lingard et al 2012; Rice et al 2010; Reeves \& Lewin 2004; Wittenberg-Lyles et al 2010). Their observations of the interactions between different professionals within a healthcare context may have overcome the challenge of access to a wider representative sample of staff. However, as reinforced by Reeves et al., (2013) and Zwarenstein et al., (2009), further high quality rigorous IPE and IPW research is required to strengthen IPW and IPE evidence.

\section{Limitations}

Two main limitations are noted in relation to the search strategy employed in this review. Firstly, by electing to focus on literature published from 2000, in line with the previously noted rise in the profile of IPE and IPW, research that pre-dated this time has been excluded. Secondly, while the search terms and keywords may have accounted for some of the interchangeable terms used to describe IPE and IPW, the search strategy did not account for variations in the term 'attitudes'.

\section{Concluding comments}

There is limited evidence related to staff attitudes to IPW and the value placed on IPE for students learning in healthcare setting. Although ths review identified four studies which included staff attitudes to IPW and IPE, only one study related to health and social care staff attitudes to IPW and their attitudes to IPE for students learning in the healthcare setting.

In relation to all of the studies reviewed, professional background and prior experience of IPE were identified as two of the main factors effecting attitudes to IPW or IPE. Other possible influencing factors such as age, gender, professional 
experience and income were considered in some of the studies, although the strength of these correlations were varied and inconsistent.

Health and social care staff play a primary role in ensuring the provision of safe, quality care to patients and carers. In their remit as workplace mentors, they are key role models for students. It is important that students have positive and influential experiences in relation to the impact of IPW on patient care and that IPE is valued as a way of preparing students for future collaborative working. In light of the restructuring of health and social care services in the UK, further research is required to investigate attitudes to IPW and IPE within a healthcare setting. It is important that this research is carried out within a relevant context and that the chosen research design suits this context.

\section{Declaration of interest}

The authors report no declarations of interest. The authors were responsible for the writing and content of this paper. 


\section{References}

Abramson, J, S., \& Mizrahi, T. (2003). Understanding collaboration between social workers and physicians: Application of a typology. Social Work in Health Care, 37, 71-100.

Anderson, E., Manek, N., \& Davidson, A. (2006). Evaluation of a model for maximizing interprofessional education in an acute hospital. Journal of Interprofessional Care, 20, 182-194.

Anderson, E. S., Cox, D., \& Thorpe, L. N. (2009). Preparation of educators involved in interprofessional education. Journal of Interprofessional Care, 23, 81-94.

Anderson, E. S., \& Thorpe, L. N. (2010). Interprofessional educator ambassadors: An empirical study of motivation and added value. Medical Teacher, 32, 11 e492-e500.

Anderson, E. S., Thorpe, L. N., \& Hammick, M. (2011). Interprofessional staff development: Changing attitudes and winning hearts and minds. Journal of Interprofessional Care, 25, 11-17.

Association of Directors of Social Work Report. (2013). Deliverying integrated care and support. Institute for Research and Innovation in Social Services.

Retrieved from http://www.socialworkscotland.org/what-we-do/Publications/

Azjen, I., \& Fishbein, M. (1977). Attitude-behaviour relations: A theoretical analysis and review of empirical research. Psychological Bulletin, 8, 888-918.

Bailey, P., Jones, L., \& Way, D. (2006). Family physician/nurse practitioner: Stories of collaboration. Journal of Advanced Nursing, 53, 381-391.

Baker, L., Egan-Lee, E., Martimianakis, M.A., \& Reeves, S. (2011). Relationships of power: implications for interprofessional education. Journal of Interprofessional Care, 25, 98-104.

Barr, H., Helme, M. and D'Avray, L. (2011). Developing interprofessional education in health and social care courses in the United Kingdom. HSAP Repository, November. Retrieved from http://repos.hsap.kcl.ac.uk/content/m10247/latest/

Barr, H., \& Low. (2013). Introducing interprofessional education. Fareham, UK: CAIPE

Baxter, S. K., \& Brumfitt, S. M. (2008). Professional differences in interprofessional working. Journal of Interprofessional Care, 22, 239-251.

Bem, D. J. (1970). Beliefs, attitudes and human affairs. Belmont, CA: Brooks/Cole Pub

Braithwaite, J., Westbrook, M., Nugus, P., Greenfield, D., Travaglia, J., Runciman, W., . . Westbrook, J. (2012). A four-year, systems-wide intervention promoting interprofessional collaboration. BMC Health Services Research, 12, 99. 
Braithwaite, J., Westbrook, M., Nugus, P., Greenfield, D., Travaglia, J. Runciman, W., ... Westbrook, J. (2013) Continuing differences between health professions' attitudes: The saga of accomplishing systems-wide interprofessionalism. International Journal for Quality in Health Care, 25, 8-15.

Chang, W., Ma, J., Chiu, H., Lin, K., \& Lee, P. (2009). Job satisfaction and perceptions of quality of patient care, collaboration and teamwork in acute care hospitals. Journal of Advanced Nursing, 65,1946-1955.

Colyer, H. M. (2008). Embedding interprofessional learning in pre-registration education in health and social care: Evidence of cultural lag. Learning in Health and Social Care, 7,126-133.

Costa, D.K., Barg, F.K., Asch, D.A., \& Kahn, J.M. (2014). Facilitators of an interprofessional approach to care in medical and mixed medical/surgical ICUs: A multicentre qualitative study. Research in Nursing \& Health, 37, 326-335.

Curran, V. R., Sharpe, D., \& Forristall, J. (2007). Attitudes of health sciences faculty members towards interprofessional teamwork and education. Medical Education, 41, 892-896

Egan-Lee, E., Baker, L., Tobin, S., Hollenberg, E., Dematteo, D., \& Reeves, S. (2011). Neophyte facilitator experiences of interprofessional education:implications for faculty development. Journal of Interprofessional Care, 25, 333-338.

Festinger, L. (1957). A theory of cognitive dissonance. Stanford, CA: Stanford University Press.

Francis, R. (2013). Report of the Mid Staffordshire NHS Foundation Trust public inquiry. Executive summary. Retrieved from http://www.midstaffspublicinquiry.com/report.

Freeth, D., Hammick, M., Reeves, S., Koppel, I., \& Barr, H. (2005). Effective interprofessional education: Development, delivery and evaluation. Oxford, UK: Blackwell Publishing Ltd.

General Medical Council. (2009). Tomorrow's doctors. Outcomes and standards for undergraduate medical education. London, UK: Author.

Gibbon, B., Watkins, C., Barer, D., Waters, K., Davies, S., Lightbody, L., \& Leathley, M. (2002). Can staff attitudes to team working in stroke care be improved? Journal of Advanced Nursing, 40,105-111.

Gordon, S. (2012). On teams, teamwork and team intelligence. In R. Koppel \& S. Gordon (Eds.) First do less harm: Confronting the inconvenient problems of patient safety. (pp196-220) New York City, NY: Cornell University Press. 
Hawker, S., Payne, S., Kerr, C., Hardey, M., \& Powell, J. (2002). Appraising the evidence: Reviewing disparate data systematically. Qualitative Health Research, 12, 1284-1299.

Health and Social Care Act. (2012). Retrieved from http://www.legislation.gov.uk/ukpga/2012/7/pdfs/ukpga_20120007_en.pdf

Health and Social Care Professions Council. (2009). Standards of education and training guidance. Retrieved from http://www.hpcuk.org/aboutregistration/standards/sets/

Herbert, C. P., Bainbridge, L., Bickford, J., Baptiste, S., Brajtman, S., Dryden, T., . . . Solomon, P. (2007). Factors that influence engagement in collaborative practice: How eight health professionals became advocates. Canadian Family Physician, 53, 1318-1325.

Hughes, C, M., \& McCann, S. (2003). Perceived interprofessional barriers between community pharmacists and general practitioners: A qualitative assessment. British Journal of General Practice, 53, 600-606.

Ireland, J., Gibb, S., \& West, B. (2008). Interprofessional education: Reviewing the evidence. British Journal of Midwifery, 16, 446-453.

Jové, A. M., Fernández, A., Hughes, C., Guillén-Solá, M., Rovira, M., \& RubioValera, M. (2014). Perceptions of collaboration between general practitioners and community pharmacists:Findings from a qualitative study based in Spain. Journal of Interprofessional Care. 28, 352-357.

Kenaszchuk, C., Conn, L.G., Dainty, K., McCarthy, C., Reeves, S., \& Zwarenstein, M. (2012). Consensus on interprofessional collaboration in hospitals: statistical agreement of ratings from ethnographic fieldwork and measurement scales. Journal of Evaluation in Clinical Practice, 18, 93-99.

Kennedy, I. (2001). Learning from Bristol: The report of the public inquiry into children's heart surgery at the Bristol Royal Infirmary 1984-1995. London, UK: Her Majesty's Stationery Office.

Kraus, S. J. (1995) Attitudes and the prediction of behaviour: A meta-analysis of the empirical literature. Personality and Social Psychology Bulletin, 21, 58 -75.

Kvarnström, S. (2008). Difficulties in collaboration: A critical incident study of interprofessional healthcare teamwork. Journal of Interprofessional Care, 22,191-203.

Laming, H. (2003). The Victoria Climbié Inquiry Report. Retrieved from http://www.publications.parliament.uk/pa/cm200203/cmselect/cmhealth/570/ 570.pdf

Larkin, C., \& Callaghan, P. (2005) Professionals' perceptions of interprofessional working in community mental health teams. Journal of Interprofessional Care, 19, $338-346$. 
Lindbland, K., Kjellgren, K.I., Ring, L., Maroti, M., \& Serup, J. (2006). The role of dermatologists, nurses and pharmacists in chronic dermatological treatment: Patient and provider views and experiences. Acta Dermato-Venereologica, 86, 202-208.

Lingard, L., McDougall, A., Levstik, M., Chandok, N., Spafford, M. M., \& Schryer, C. (2012). Representing complexity well: A story about teamwork, with implications for how we teach collaboration. Medical Education, 46, 869-877.

Matziou, V., Vlahioti, E., Perdikaris, P., Matziou, T., Megapanou, E., \& Petsios, K. (2014). Physician and nursing perceptions concerning interprofessional communication and collaboration. Journal of Interprofessional Care, 28, 526533.

McCray, J. (2003). Interprofessional practice and learning disability nursing. British Journal of Nursing, 12, 1335-1344.

Moher, D., Liberati, A. Tetzlaff, J., \& Altman, D.G. (2009). Preferred Reporting Items for Systematic Reviews and Meta-Analyses: The PRISMA Statement. PLoS Med 6(6): e1000097.doi:101371/journal.pmed1000097

Nursing and Midwifery Council. (2010). Standards for pre-registration nursing education. London, UK: Author.

O'Carroll,V., Braid, M., Ker, J., \& Jackson, C. (2012). How can student experience enhance the development of a model of interprofessional clinical skills education in the practice placement setting? Journal of Interprofessional Care, 26, 508-10.

O'Carroll,V., Smith,J., Collinson, A., Jackson, C., \& Ker, J. (2013). Interprofessional education for students' in primary care placements: Widening opportunities for accessible interprofessional learning. Medical Science Educator, 23 (special issue), 405-410.

Piquette, D., Reeves, S., \& Leblanc,V.R. (2009). Interprofessional intensive care unit team interactions and medical crisis: a qualitative study. Journal of Interprofessional Care, 23, 273-285.

Pollard, K. C., \& Miers, M. E. (2008). From students to professionals: Results of a longitudinal study of attitudes to pre-qualifying collaborative learning and working in health and social care in the United Kingdom. Journal of Interprofessional Care, 22, 399-416.

Pollard, K. C., Miers, M. E., \& Rickaby, C. (2012). "Oh why didn't I take more notice?" Professionals' views and perceptions of pre-qualifying preparation for interprofessional working in practice. Journal of Interprofessional Care, 26,355-361.

Powell, A.E., \& Davies, H, T.O. (2012). The struggle to improve patient care in the face of professional boundaries. Social Science \& Medicine, 75, 807-814. 
Rees, D., \& Johnson, R. (2007). All together now? Staff views and experiences of a pre-qualifying interprofessional curriculum. Journal of Interprofessional Care, $21,543-555$.

Reeves, S., Goldman, J., Gilbert, J., Tepper, J., Silver, I., Suter, E., \& Zwarenstein, M., (2011). A scoping review to improve conceptual clarity of interprofessional interventions. Journal of Interprofessional Care. 25, 167-174.

Reeves, S., \& Lewin, S. (2004). Interprofessional collaboration in the hospital: Strategies and meanings. Journal of Health Services Research and Policy, 9, 218-225.

Reeves, S., Perrier, L., Goldman, J., Freeth, D., \& Zwarenstein, M. (2013). Interprofessional education: Effects on professional practice and healthcare outcomes (Update). The Cochrane Library. Issue 3, West Sussex, UK:John Wiley \& Sons, Ltd.

Reid, R., Bruce, D., Allstaff, K., \& McLernon, D. (2006). Validating the readiness for interprofessional learning scale (RIPLS) in the postgraduate context: Are healthcare professionals ready for IPL? Medical Education, 40, 415-422.

Rice, K., Zwarenstein, M., Conn, L.G., Kenaszchuk, C., Russell, A., \& Reeves, S. (2010). An intervention to improve interprofessional collaboration and communications: a comparative qualitative study. Journal of Interprofessional Care, 24, 350-361.

Robben, S., Perry, M., van Nieuwenhuijzen, L., van Achterberg, T., Rikkert, M. O., Schers, H., .. .Melis, R. (2012). Impact of interprofessional education on collaboration attitudes, skills, and behavior among primary care professionals. The Journal of Continuing Education in the Health Professions, 32, 196-204.

Russell, L., Nyhof-Young, J., Abosh, B., \& Robinson, S. (2006). An exploratory analysis of an interprofessional learning environment in two hospital clinical teaching units. Journal of Interprofessional Care, 20, 29-39.

Snelgrove, S., \& Hughes, D. (2000). Interprofessional relations between doctors and nurses: Perspectives from South Wales. Journal of Advanced Nursing, 31, 661-667.

Stake, R. E. (2005). The art of case study research. London,UK: Sage Publications Ltd.

Stew, G. (2005). Learning together in practice: A survey of interprofessional education in clinical settings in South-East England. Journal of Interprofessional Care, 19, 223-235.

Suter, E., Arndt, J., Arthur, N., Parboosingh, J., Taylor, E., \& Deutschlander, S. (2009). Role understanding and effective communication as core competencies for collaborative practice. Journal of Interprofessional Care, 23, 41-51. 
The Joint Commission. (2013). Sentinel event data root causes by event type 2004 June 2013. Retrieved from

http://www.jointcommission.org/assets/1/18/Root_Causes_by_Event_Type_ 2004-2Q2013.pdf

Wittenberg-Lyles, E., Oliver, D. P., Demiris, G., \& Regehr, K. (2010). Interdisciplinary collaboration in hospice team meetings. Journal of Interprofessional Care, 24, 264-273.

World Health Organisation. (2010) Framework for action on interprofessional education and collaborative practice. WHO, Switzerland. Retrieved from http://www.who.int/hrh/nursing_midwifery/en/.

Yin, R. K. (2003). Case study research. Design and methods. ( $3^{\text {rd }}$ ed.) Thousand Oaks, CA: Sage Publications, Inc.

Zwarenstein, M., Goldman, J., \& Reeves, S. (2009). Interprofessional collaboration: Effects of practice-based interventions on professional practice and healthcare outcomes (Review). The Cochrane Library. Issue 3. West Sussex, UK:John Wiley \& Sons, Ltd. 